\title{
THE TIME COURSE OF ADAPTATION TO SPATIAL CONTRAST
}

\author{
Mark W. Greenlee, ${ }^{1 *}$ Mark A. Georgeson, ${ }^{2}$ Svein Magnussen ${ }^{3}$ and John P. Harris ${ }^{2}$ \\ 'Neurologische Universitätsklinik mit Abteilung für Neurophysiologie, Hansastrasse 9, 7800 Freiburg, \\ F.R.G., ${ }^{2}$ Department of Psychology, University of Bristol, Bristol, U.K. and ${ }^{3}$ Institute of Psychology, \\ University of Oslo, Oslo. Norway
}

(Received 17 October 1989; in revised form 23 May 1990)

\begin{abstract}
We explored the buildup and decay of threshold elevation during and after adaptation to sinewave gratings in a series of experiments investigating the effects of adapting time, adapting contrast, spatial frequency and retinal eccentricity. Contrast thresholds for vertical sinewave gratings truncated in space by a one-dimensional Gaussian envelope were measured before and after adaptation to a full-field suprathreshold grating of the same spatial frequency and orientation. Thresholds were measured intermittently after adaptation in a "seen/not-seen" single presentation procedure until these thresholds returned to baseline values. The first test grating was presented $300 \mathrm{msec}$ after the offset of the adapting stimulus, and thereafter at regular intervals. At different times after adaptation, contrast thresholds were estimated by off-line analysis of the data using the QUEST algorithm. Adapting time was either 1, 10, 100 or $1000 \mathrm{sec}$ and adapting contrast was either $9,19,29$ or $39 \mathrm{~dB}(\mathrm{re}, 1 \%)$. The test gratings were presented centered either at the fixation point or at 5 and $10 \mathrm{deg}$ eccentricity along the horizontal meridian. The results suggest that up to the saturation level the buildup and the decay of adaptation to contrast is well described by a power function of time. The slope of the best fitting line on log-log axes is fairly constant for the adaptation times tested. As reported earlier, thresholds increased with adapting contrast and these contrast-dependent differences were evident $300 \mathrm{msec}$ after the termination of adaptation. Adaptation at $10 \mathrm{deg}$ eccentricity yielded slightly higher threshold elevations than for central vision. Based on these results, a description is given of the dynamic response of the underlying neural mechanisms.
\end{abstract}

Contrast adaptation Gain control Contrast thresholds Retinal eccentricity

\section{INTRODUCTION}

Viewing of a high-contrast grating elevates contrast thresholds for subsequently detecting gratings of similar spatial frequency and orientation (Blakemore \& Campbell, 1969; Pantle \& Sekuler, 1968; Sekuler \& Ganz, 1963). The elevation in contrast threshold following adaptation has been shown to depend on the spatial frequency (Blakemore \& Campbell, 1969; Pantle \& Sekuler, 1968) and orientation (Blakemore \& Nachmias, 1971) difference between the adapting and test gratings. The magnitude of this threshold elevation further depends on the adapting contrast (Björklund \& Magnussen, 1981; Blakemore \& Campbell, 1969; Georgeson \& Harris, 1984) and the duration of adaptation (Björklund \& Magnussen, 1981; Blakemore \& Campbell, 1969; Magnussen \& Greenlee, 1985; Rose \& Evans, 1983). Although it was originally suggested that the effect of adaptation to contrast saturates after as little as $\mathbf{4 0 ~} \mathrm{sec}$ (Blakemore

*To whom correspondence should be addressed.
\& Campbell, 1969), Magnussen and Greenlee (1985) demonstrated that, for an adapting contrast of 0.6 , thresholds continue to rise for up to 30-60 min of adaptation for different subjects. The time course of the dynamic range of the buildup and decay of this adaptation was found to be best fitted by a power function (i.e. linear on axes of log contrast threshold vs log adapting time).

Earlier studies of the buildup and decay of contrast adaptation have used the adjustment method to determine thresholds during and after adaptation (e.g. Blakemore \& Campbell, 1969; Magnussen \& Greenlee, 1985; Rose \& Evans, 1983). Daugman (1983) has argued that tracking contrast thresholds with the method of adjustment is contaminated by the response time required for the observer to make a setting. In a two-interval forced-choice paradigm, Daugman (1983) reported much shorter decay constants than had previously been found. In a more recent study, Lorenceau (1987) used a threshold tracking procedure which combined reaction time measurement and adjustment methods. 
After $60 \mathrm{sec}$ adaptation to a high-contrast grating, he presented a lower contrast test grating and recorded the time at which the subject first detected and then responded to the grating. The contrast was subsequently lowered until the next response was recorded and so on. In the final stage of recovery from adaptation, the method of adjustment was used. He found contrast threshold elevation to be best fitted by an exponential function of recovery time (Lorenceau, 1987, Table 2). However, if only the dynamic portion of the decay function is considered by excluding the thresholds measured with the adjustment method then a power function provides a good fit to the data (his Fig. 4, ). Ho and Berkley (1988) have also argued that an exponential function best fits the decline in VEP amplitude during the presentation of high-contrast, phase-reversing gratings.

\section{Rationale of the present imvestigation}

The present study attempts to investigate more fully the buildup and decay of contrast adaptation. Georgeson and Georgeson (1987) have argued that the aftereffects of forward masking and prolonged adaptation result from the same neural process, the only difference being that, compared to the effects of adaptation, the effects of forward masking are short-lived. Following $25 \mathrm{msec}$ exposure to the forwardmasking stimulus, thresholds are elevated for 100-200 msec. The initial effect of brief forward masking is, however, according to Georgeson and Georgeson (1987) as large as the effect of prolonged adaptation. On this view, the apparent buildup of adaptation with time shown by Magnussen and Greenlee (1985) reflects the fact that they made their first measurement approx. 6 sec after the end of adaptation. The aftereffect from short adapting times would decay faster than those from longer adapting times. Thus the adaptation would appear to build up for longer adapting times, even though the immediate postadaptation threshold elevation was the same for all adapting times. To explore the time course of the buildup and decay of adaptation, we introduce a method in which test gratings of specified contrast were presented at known times after the end of adaptation so that threshold measurements were not contaminated by reaction time. In the experiments to be reported, the first test grating was presented $300 \mathrm{msec}$ after the offset of the adapting stimulus and then at regular intervals until thresholds had returned to preadaptation values. In this manner we could probe the early and long-lasting effects of adaptation using the same method of threshold determination. Based on this technique, we explore the effects of adapting time, adapting contrast, spatial frequency and retinal eccentricity on the buildup and decay of adaptation. The results permit a comprehensive description of the process of contrast adaptation.

\section{METHOD}

\section{Apparatus}

Sinusoidal luminance gratings were generated on a high-resolution CRT (Joyce Electronics, Cambridge, U.K.). The display had a white (P4) phosphor, a frame rate of $100 \mathrm{~Hz}$, and a spaceaveraged luminance of $200 \mathrm{~cd} / \mathrm{m}^{2}$. Analog signals under the control of a microprocessor determined the position, spatial frequency, spatial phase, temporal frequency and contrast of the grating stimuli. The display was viewed binocularly with natural pupils at a distance of $114 \mathrm{~cm}$, at which distance the screen subtended $11 \times 15$ deg visual angle. Viewing distance and head orientation were held constant by a chinforehead rest. The display was surrounded by a back-illuminated white Plexiglass screen, the mean luminance and color temperature of which were adjusted to closely match that of the CRT. The mean luminance and contrast of the display were checked regularly with a spot photometer. To aid fixation, a small (o.d. $18 \mathrm{~min}$ arc) highcontrast, Letraset circle was attached to the center of the display. The fixation circle also enabled subjects to check their accommodation during adaptation and testing. The experimental room was otherwise dimly lit.

\section{Stimuli}

A full-field $(11 \times 15 \mathrm{deg})$, vertically oriented, sinusoidal adapting grating was presented for a duration of $1,10,100$ or $1000 \mathrm{sec}$. The spatial phase of the adapting grating reversed as a sinusoidal function of time at a rate of $5 \mathrm{~Hz}$. Most of the experiments are based on measurements following adaptation to a grating with $39 \mathrm{~dB}(89.1 \%)$ contrast (contrast is expressed in $\mathrm{dB}$ relative to $1 \%$ ). In the experiment concerning the effect of adapting contrast on threshold elevation, the contrast of the adapting gratings was varied in $10 \mathrm{~dB}$ steps from 9 to $39 \mathrm{~dB}$. The first set of experiments was conducted using a full-field test grating. All test gratings were sinusoidally flickered at $5 \mathrm{~Hz}$. This means that the spatial phase of the test grating changed by 
$180 \mathrm{deg}$ phase angle during the $100 \mathrm{msec}$ presentation. In the experiments exploring the effect of adapting contrast, spatial frequency and retinal eccentricity, we used test gratings whose contrast was tapered along the horizonal meridian by a Gaussian envelope. The standard deviation of the one-dimensional Gaussian envelope was adjusted for each spatial frequency so that the resulting Gabor test grating had a constant bandwith of 0.46 octave ( $\mathrm{SD}=1.2$ periods of grating; cf. Kulikowski, Marcelja \& Bishop, 1982). Thus the same number of periods fell under the contrast envelope independent of the spatial frequency of the grating. The contrast of the test gratings was turned on and off as a rectangular function of time and the stimulus duration was $100 \mathrm{msec}$ in all experiments. Thresholds measured after adaptation to a high-contrast grating are compared with those measured after adaptation to a blank field of the same mean luminance (referred to in the following sections as baseline values).

\section{Procedure}

The observer initiated a recording session by pressing one of two buttons on the response box. This started the adaptation period, in which either a blank field or a high-contrast grating flickering at $5 \mathrm{~Hz}$ was presented. During adaptation, the subject fixated the Letraset circle at the center of the display. After termination of the adapting stimulus, a brief, computer-generated tone cued the subject that the test period was to begin. Each subsequent test grating was preceded by such a tone approx. $200 \mathrm{msec}$ before stimulus onset. The first test grating was presented $300 \mathrm{msec}$ after the offset of the adapting grating. The observer responded by pressing one of two buttons, to indicate whether he "saw" the grating or not. As all the observers participating in the experiments have had considerable practice in such tasks, and since thresholds over a number of practice measurements in the unadapted state prior to data collection showed a very small variation, we believe that the observer's criterion for his "yes-no" judgment did not vary significantly in the experiments. After each "yes" response, test contrast was decremented by $3 \mathrm{~dB}$, and after each "no" response test contrast was incremented by $2 \mathrm{~dB}$. In pilot work, we found that during the first $5 \mathrm{sec}$ after adaptation contrast thresholds fell at a rate greater than $3 \mathrm{~dB}$ in successive response intervals. Thus the program controlling the experiment reduced contrast by $5 \mathrm{~dB}$ following a "yes" response if the stimulus was presented within $5 \mathrm{sec}$ of the end of adaptation. The initial contrast levels of the test grating were based on preliminary estimates (or the best guess) of the contrast level at which the initial threshold was located. Five different initial contrast levels, centered around this estimate and differing by $2 \mathrm{~dB}$ each, were presented on separate runs. The observer's "yes-no" responses were recorded by the computer together with the time after adaptation at which the stimulus was presented. 5-10 repetitions of each condition were conducted for each observer. These data were subsequently analyzed off-line. The response data were sorted into time bins. The width of each time bin was approximately the same on a log scale with respect to the time after adaptation. Thus the first 5 time bins had a width of $1.2 \mathrm{sec}$ and the subsequent bins had a width of $0.2 \mathrm{log} \mathrm{sec}$. The bin width thus varied from $1.2 \mathrm{sec}$ for a stimulus given up to $6 \mathrm{sec}$ after adaptation to $294 \mathrm{sec}$ for a stimulus presented $500 \mathrm{sec}$ after adaptation. As the longest interval between test stimuli used was $10 \mathrm{sec}$, many more observations fell within the later time bins. However, the change in threshold occurring within a single bin remained approximately constant over bins, since the decay of adaptation can be approximated by a power function of time (Magnussen \& Greenlee, 1985). It should be emphasized here that it was the time of presentation of the test grating rather than the time at which the subject responded which decided in to which time bin a response was placed. Although most responses occurred within $300-400 \mathrm{msec}$ of the stimulus onset, a late response was also counted and placed in the bin for that stimulus onset time. In the worse case, a late response delayed the next stimulus, thus causing one time bin to be skipped. As the subjects were highly motivated, such late responses were very infrequent.

\section{Data analysis}

Thresholds for each time bin were estimated to the nearest $0.5 \mathrm{~dB}$ by applying off-line the maximum-likelihood procedure (QUEST) developed by Watson and Pelli (1983). The method assumes that the psychometric function is well fitted by the following Weibull function:

$p(c)=1-(1-g) * \exp \left\{-\left[10^{(b / 20)(c+e-T)}\right]\right\} ;$

where $p(c)$ is the probability of a "yes" response at test contrast $c$ (in $\mathrm{dB}$, re. $1 \%$ ), $\mathrm{T}=$ threshold in $\mathrm{dB}$ and $e, g$ and $b$ are constants. The value of $e(-0.965)$ determines the threshold criterion 
(here $50 \%$ "yes" responses), $g$ is the falsepositive rate, and $b$ controls the slope of the psychometric function. In the control conditions the data are fitted reasonably well by the values $g=0.05$ and $b=4$ (see Fig. 1). For the data measured after adaptation the QUEST estimation procedure was applied, since there were not enough observations within each bin to allow a psychometric function to be plotted. The threshold value $(T)$ is computed as that which maximizes the likelihood of obtaining the set of responses actually observed. The QUEST method also yields confidence limits for each threshold estimate, allowing the rejection of occasional points where the estimates were grossly unreliable. It has been suggested that the slope of the psychometric function is steeper following adaptation (Williams \& Wilson, 1983). We re-analyzed some of our data with $b=8$ instead of $b=4$. Threshold estimates generally did not change by more than $0.5 \mathrm{~dB}$ after such an alteration, suggesting that the method is robust with respect to changes in the slope parameter.

The effects of adapting time and adapting contrast on initial threshold elevation were tested using analysis of variance techniques (BMDP program IV, version 4.85, VAX/VMS). The effects of adapting time and adapting contrast on the slope of recovery from adaptation were tested using regression analysis and a test for the reduction of residuals by grouping (BMDP program 1R, version 4.85, VAX/VMS; reduction of residuals due to grouping). A significant $F$-ratio here would indicate that the slope coefficients of the regression equations differ across the grouping variable.

\section{Subjects}

The observers were the four authors, all of whom had corrected-to-normal visual acuity. A minimum of two observers were tested for each of the conditions examined. No substantial individual differences in initial thresholds or adaptation levels could be detected.

\section{RESULTS}

Psychometric functions obtained after adaptation to a blank field are shown by solid symbols in Fig. 1 for subjects MAG and MWG. These results are compared with those measured after the subjects had recovered from adaptation (open symbols). As both data sets are fit reasonably well by the same Weibull function described above, the underlying pyschometric function appears to be fairly stable over time.

\section{Contrast threshold elevation and adapting time}

Figure 2 presents the findings of the experiment in which we investigated the effect of adapting time on the initial threshold elevation and the recovery from adaptation. The spatial frequency of the adapting and test grating was $3 \mathrm{c} / \mathrm{deg}$ and adapting contrast was $39 \mathrm{~dB}$. Contrast threshold (in $\mathrm{dB}$ ) is plotted as a function of the logarithm of recovery time (in sec). Results for MAG are depicted in Fig. 2a and those from MWG in Fig. 2b. The different symbols represent the thresholds estimated by the QUEST procedure after $1(\bigcirc), 10(O)$, $100(\square)$ and $1000(\square)$ sec adaptation. The slopes of the decay functions are well approximated by straight lines on $\log$ - $\log$ coordinates.

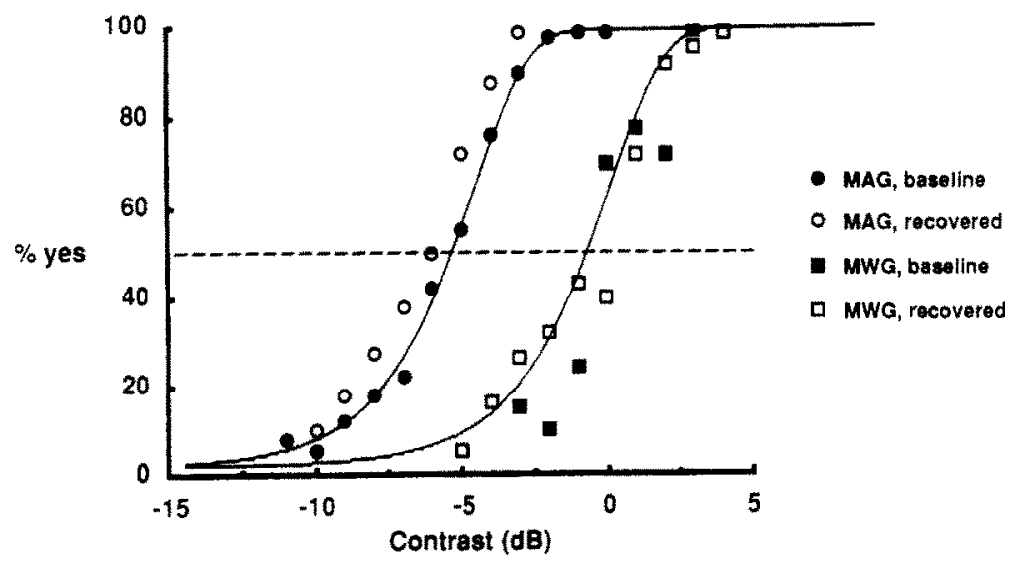

Fig. 1. Psychometric functions for detecting a $3 \mathrm{c} / \mathrm{deg}$ sinewave gratings for subjects $M A G$ and $M W G$ after adaptation to a blank screen (zero contrast, solid symbols) and after recovery from adaptation to a high-contrast grating (open symbols). The curves are the Weibull functions described in the text. Note that data from MWG have been arbitrarily shifted to the right by $5 \mathrm{~dB}$ along the contrast axis for sake of clarity. 


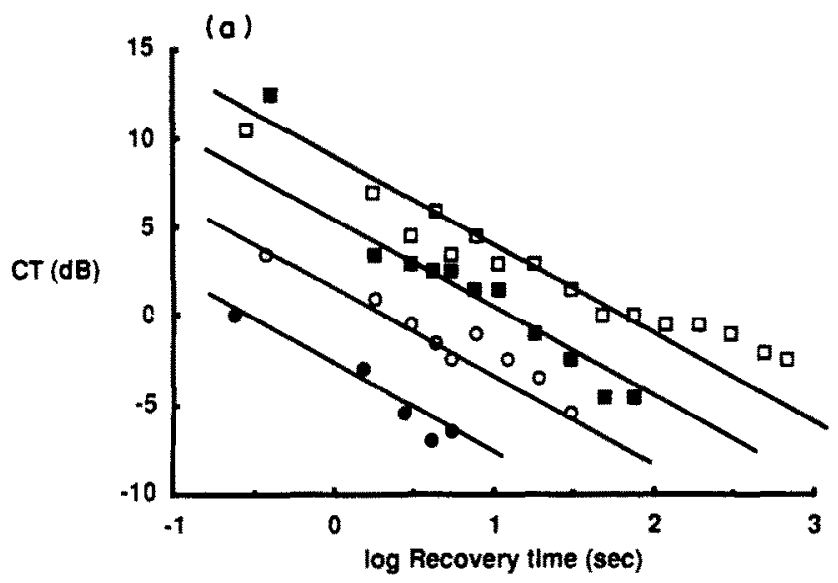

(b)

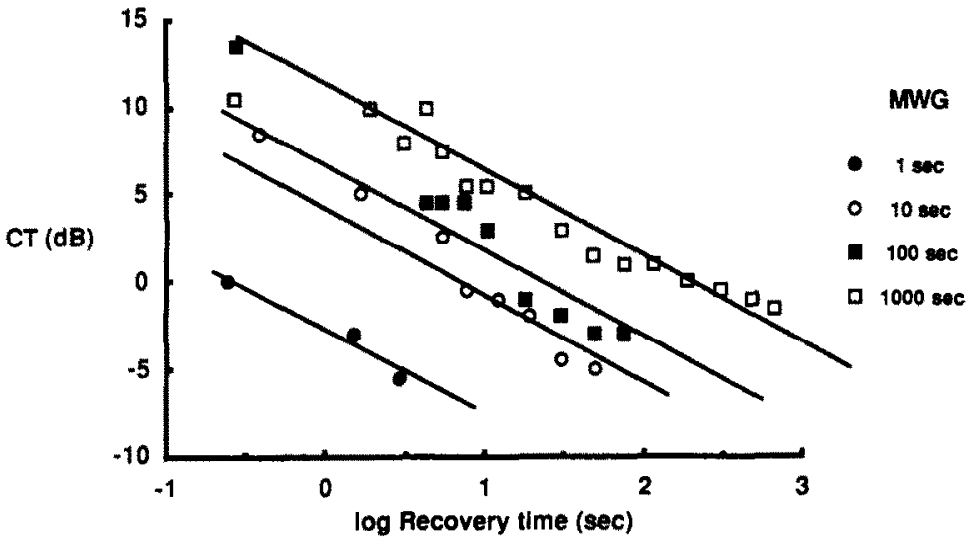

Fig. 2. Contrast threshold (in $\mathrm{dB}$ ) for detecting a $3 \mathrm{c} / \mathrm{deg}$ test grating after adaptation to a grating of the same spatial frequency and orientation with a contrast of $39 \mathrm{~dB}(89.1 \%)$ as a function of $\log$ recovery time. The different symbols represent threshold estimates made during recovery following $1(O), 10(\mathrm{O})$, 100 (O) and 1000 (口) sec adaptation. Values for observer MAG are shown in (a) and those for MWG in (b). The lines represent the linear regression of log contrast threshold on log recovery time. For both subjects the slopes were constrained to be equal.

Supporting the earlier work by Magnussen and Greenlee (1985), contrast thresholds increased as a function of adaptation time (upward shift of regression lines in Fig. 2) and decreased as a power function of recovery time. Several linear regression models were explored. The simplest of these models is that the regression lines are parallel and are equally spaced (i.e. for each log unit increment in adaptation time the functions are shifted upwards by the same amount). For subject MAG this model gave a good fit to the data with a slope of $-4.5 \mathrm{~dB}$ per log sec time and accounts for $90.4 \%$ of the variance in the data. For MWG the same model accounted for $87.6 \%$ of the variance. Allowing unequal spacing between the regression lines for MWG's data increased the coefficient of determination $\left(r^{2}\right)$ to $92.1 \%$ with a slope of $-5.1 \mathrm{~dB}$ per log sec time. Relaxing the equal spacing constraint for MAG only increased $r^{2}$ to $91.4 \%$.
In Fig. 3 we replotted the data of Fig. 2 to demonstrate the single underlying function governing the data. Contrast threshold (in dB) is now plotted as a function of $t$ where:

$$
t=\log _{10}(\text { recovery time/adaptation duration) }
$$

i.e. the logarithm of the ratio between the time after adaptation at which threshold was tested to the time spent adapting (in sec). If increasing the duration of adaptation merely shifts the recovery function to the right on $\log -\log$ axes, then plotting the data as a function of $t$ should collapse the threshold data on to a single regression line. The least-squares regression of contrast thresholds on $t$ for all four adaptation durations accounts for $88.4 \%$ of total variance for subject MAG and $84.3 \%$ of the variance of the data for subject MWG. 

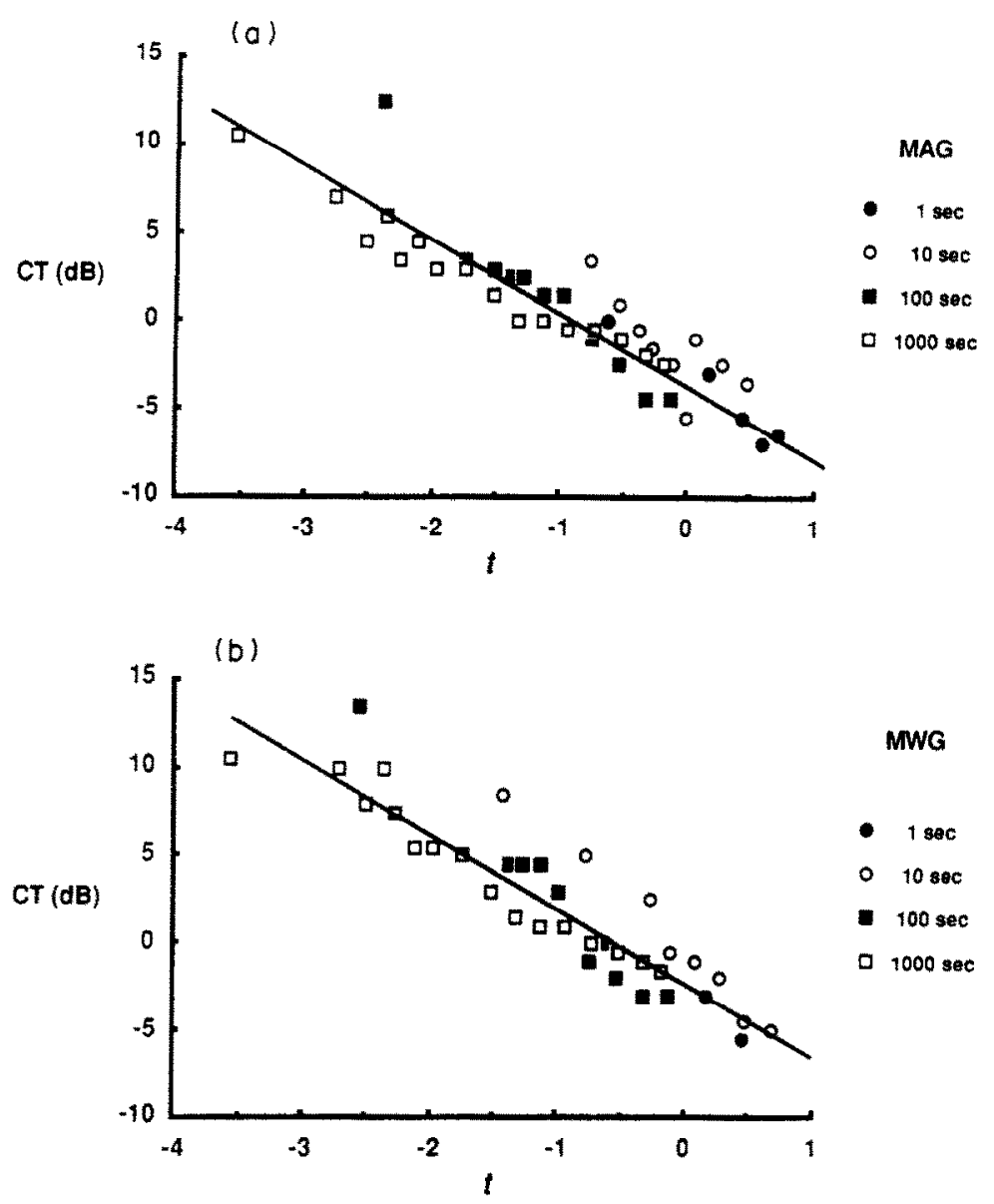

Fig. 3. A replot of the threshold estimates shown in Fig. 2, where contrast threshold is now plotted as a function of $t$, where $t=\log _{10}$ (recovery time/adapting time). For more details see text. Results for observer MAG are shown in (a) and those for MWG in (b).

Contrast threshold elevation and adapting contrast

The buildup of threshold elevation has been shown to depend on the contrast of the adapting grating (Blakemore \& Campbell, 1969; Georgeson \& Harris, 1984). We next investigated how adapting contrast affects initial contrast threshold estimates and the recovery from adaptation. The adapting and test gratings in these experiments had a spatial frequency of $4 \mathrm{c} / \mathrm{deg}$ and a vertical orientation. Test grating contrast was modulated along the horizontal meridian by a one-dimensional Gaussian (see Method). Adaptation time varied from 1 to $1000 \mathrm{sec}$. The test gratings were centered at the fixation point. In Fig. 4 initial contrast threshold (in dB) for detecting a $4 \mathrm{c} / \mathrm{deg}$ test grating is plotted as a function of adapting contrast (in $\mathrm{dB}$ ) for subjects MWG and JPH. As the first few test presentations following adaptation yielded the least reliable threshold measurements (owing to the small $n$ in each time bin, see Method), we plot the estimated threshold value for $1 \mathrm{sec}$ after adaptation based on the regression of contrast thresholds on recovery time. In this way, we could increase the reliability of the initial threshold estimates, especially for the longer adapting times. The different symbols present the results following $1(\mathbf{O}), 10(\mathrm{O}), 100(\mathbf{D})$, and $1000(\square)$ sec adaptation. Two aspects in the data are of interest here: first, with increasing adaptation time the threshold vs adapting contrast curves are shifted upwards by an equal amount (see also Fig. 2). Second, threshold elevation increases with increasing adapting contrast. Note that the shape of the curves is fairly independent of the adapting duration.

To further illustrate how adapting contrast and adapting time interact to determine the resultant elevation in contrast threshold, we replotted the results of Fig. 4. We now show contrast threshold estimated $1 \mathrm{sec}$ postadaptation as a function of the time spent adapting. The parameter is the contrast of the adapting grating, which varied from 9 to $39 \mathrm{~dB}$ (see inset). The results for observer MWG are shown in 

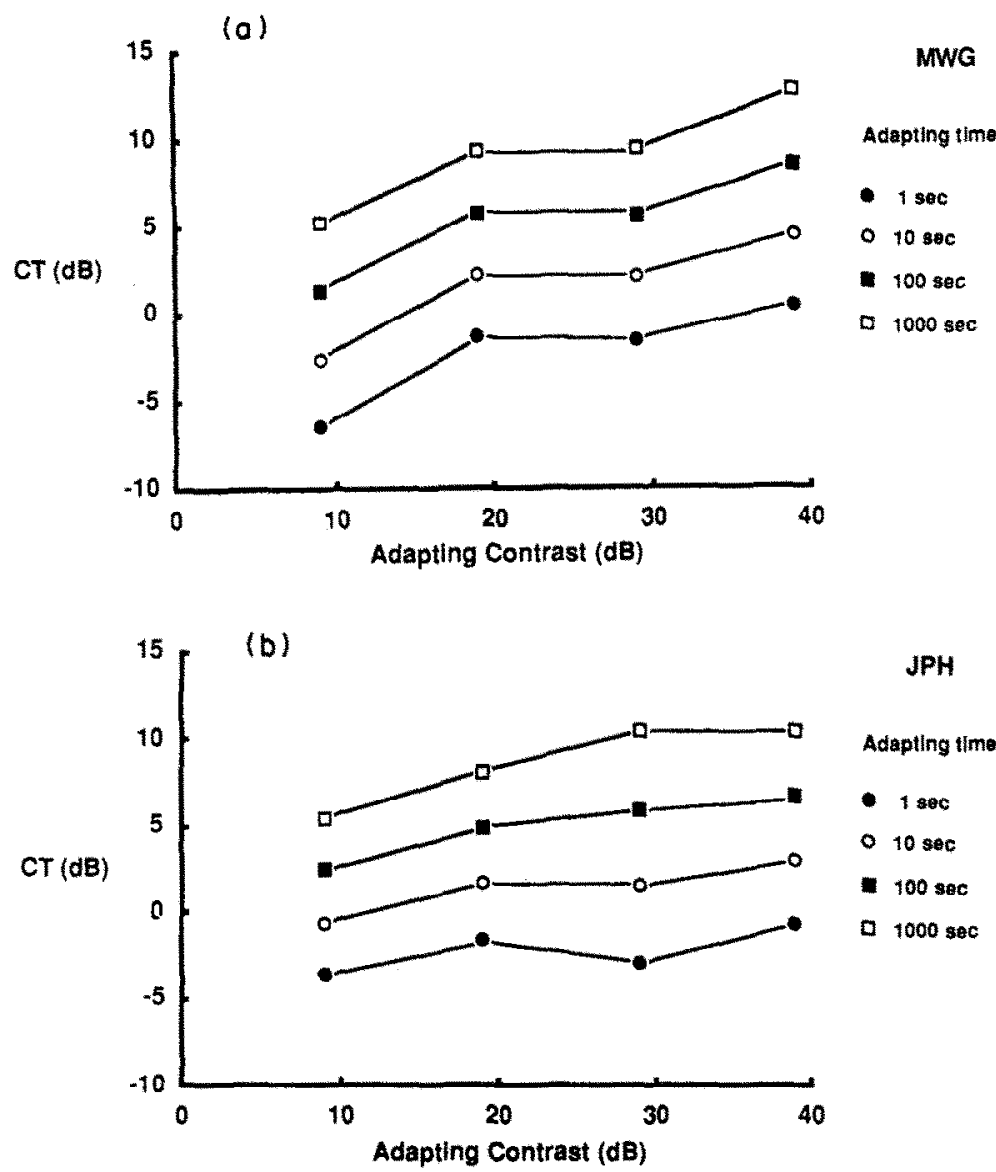

Fig. 4. Contrast thresholds for detecting a $4 \mathrm{c} / \mathrm{deg}$ grating as a function of the contrast of a $4 \mathrm{c} / \mathrm{deg}$ adapting grating. The data points represent the estimated values $1 \mathrm{sec}$ after adaptation based on the least-squares fit of the regression line describing the function contrast threshold vs recovery time. The different symbols represent the findings after $1(0), 10(0), 100(\square)$ and $1000(\square)$ sec adaptation. Results from observer MWG are shown in (a) and those from JPH in (b).

Fig. 5a and those for JPH in Fig. 5b. These results indicate that the buildup in threshold elevation is well described by a power function of adapting time (linear on $\log -\log$ axes). With only one exception (observer JPH, $29 \mathrm{~dB}$ adapting contrast), the slope of the contrast threshold vs adapting time functions was similar for all adapting contrasts, suggesting that the time course of threshold change is independent of the contrast of the adapting grating.

\section{Recovery from adaptation and adapting contrast}

We next explored the effect of adapting contrast on the time course of recovery from contrast adaptation. The results of this analysis are shown in Fig. 6 for subjects MWG (a and c) and for JPH (b and d). Panels (a) and (b) present the results for the $100 \mathrm{sec}$ adapt duration and panels (c) and (d) show the results for the $1000 \mathrm{sec}$ adapt duration. As in Fig. 3, the results have been normalized by equation (2), and con- trast threshold (in $\mathrm{dB}$ ) is plotted as a function of $t$. We constrained the regression lines shown in Fig. 6 so as to have a common intercept at the baseline contrast threshold level (indicated by horizontal, dashed line). This common intercept represents the average value of the regression coefficients. The results indicate that the slope of the decay functions vary with adapting contrast level. The time required for thresholds to reach the unadapted level remains, however, fairly constant across the different adapting contrast levels.

A two-way analysis of variance was conducted to test the statistical significance of the effects of subject, adapting contrast and adapting time on the buildup and decay of threshold elevation. The results of this analysis are presented in Table 1 . The results indicate a highly significant effect of subject, adapting time and adapting contrast on threshold elevation. There is no significant interaction between these three 


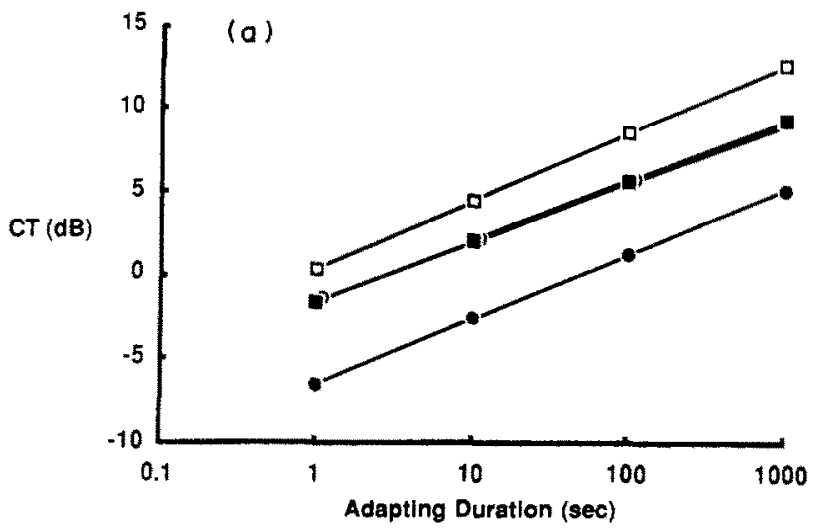

\section{MWG}

adapsing contrast

- $9 \mathrm{~dB}$

$19 \mathrm{~dB}$

- $28 \mathrm{~dB}$

口 $38 \mathrm{~dB}$

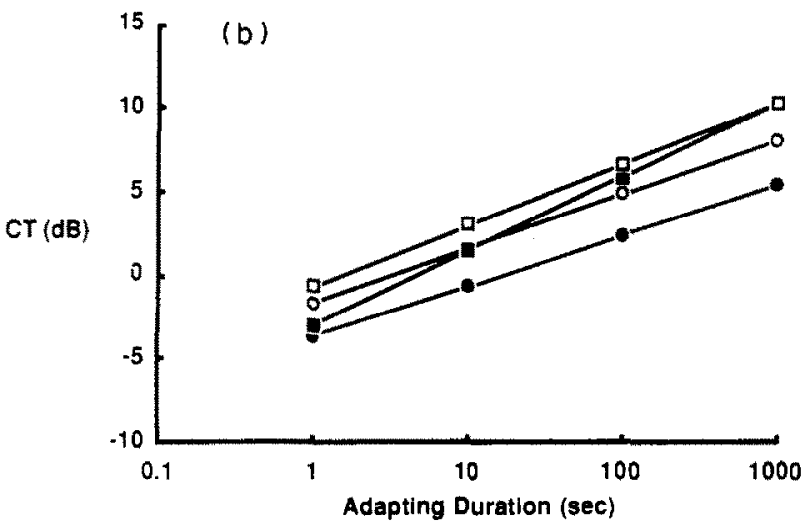

JPH

adapting contrast

- $9 \mathrm{~dB}$

C $19 \mathrm{~dB}$

- $29 \mathrm{~dB}$

व $39 \mathrm{~dB}$

Fig. 5. Contrast thresholds for detecting a $4 \mathrm{c} / \mathrm{deg}$ grating as a function of adapting duration (in sec). The data points are taken from Fig. 4 and represent the estimated values $1 \mathrm{sec}$ after adaptation based on the regression of contrast threshold on recovery time. The different symbols represent the findings after adaptation to $9(\mathbf{O}), 19(\mathrm{O}), 29(\mathbf{D})$ and $39 \mathrm{~dB}(\square)$ contrast. Results from observer MWG are shown in (a) and those from JPH in (b).

terms. A more conservative test of the effects of adapting time and adapting contrast would be to use the respective subject interaction as the error term. This analysis still indicates that the effect of adapting time $\left(F_{3,3}=130.9, P<0.01\right)$ reaches higher levels of significance than the effect of adapting contrast $\left(F_{3,3}=9.53, P<0.05\right)$. We also tested for the equality of slopes of

Table 1. Results of two-way analysis of variance of the main effects of subject, adapting time and adapting contrast on contrast thresholds, and the interactions between these main effects. The adapting and test spatial frequency was $4 \mathrm{c} / \mathrm{deg}$ and the test grating was presented centered at the point of fixation. Results from observers MWG and JPH

\begin{tabular}{lrrrc}
\hline & \multicolumn{5}{c}{ Two-way analysis of variance } \\
Source of variance & d.f. & M.S. & \multicolumn{1}{c}{$F$} & \multicolumn{1}{c}{$P$} \\
\hline Subject & 1 & 74.9 & 8.2 & 0.005 \\
Adapt time & 3 & 536.8 & 58.7 & 0.00001 \\
Adapt contrast & 3 & 62.9 & 6.9 & 0.0002 \\
Subject $\times$ adapt time & 3 & 4.1 & 0.45 & NS \\
Subject $\times$ adapt contrast & 3 & 6.6 & 0.72 & NS \\
Adapt time $\times$ adapt contrast & 3 & 8.6 & 0.94 & NS \\
Subject $\times$ adapt time $\times$ & & & & \\
$\quad$ adapt contrast & 9 & 10.2 & 1.11 & NS \\
Error & 265 & 9.1 & & \\
\hline
\end{tabular}

the functions describing contrast threshold vs $\log$ recovery time across different adapting times and adapting contrasts for each observer separately. This analysis clearly shows that the effect of adapting contrast on the slope of recovery is larger (observer MWG: $F_{9,139}=21.2$, $P<0.000005$; observer JPH; $F_{9,134}=4.56, P<$ 0.00001 ) than the effect of adapting time (observer MWG: $F_{9,139}=3.6, P<0.0005$; observer JPH: $F_{9,134}=2.7, P<0.006$ ).

\section{Effects of spatial frequency and retinal eccentricity}

In the next experiment we explored the effect of retinal eccentricity on the initial elevation in threshold and recovery from adaptation for test stimuli scaled in size to approximately compensate for the retino-cortical magnification factor (Cowey \& Rolls, 1974; Rovamo \& Virsu, 1979). Subjects adapted to a full-field adapting grating and afterwards were tested with one-dimensional Gabor test gratings centered at the fixation point or 5 and $10 \mathrm{deg}$ eccentric along the horizontal meridian (left visual field). During adaptation 

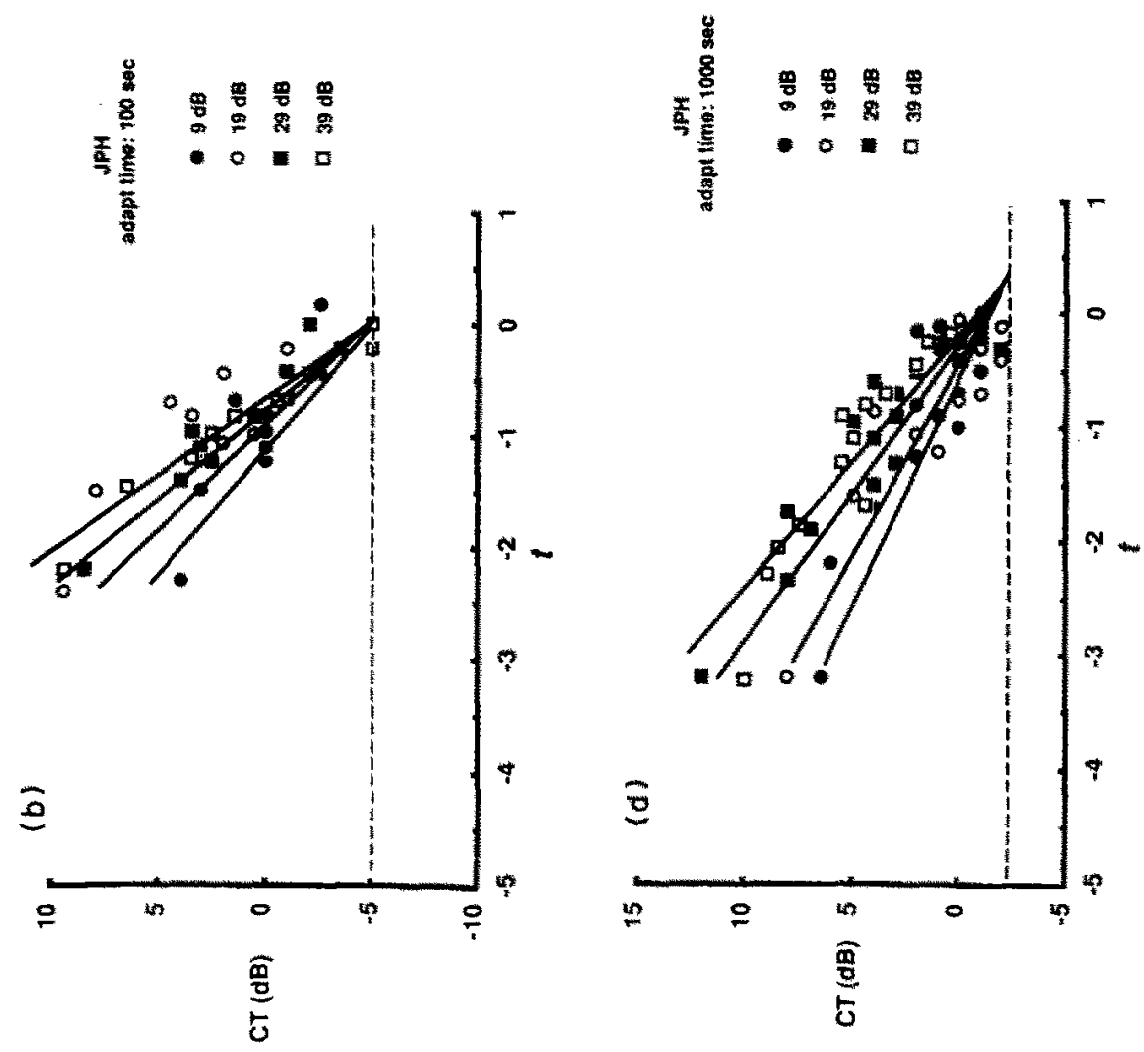

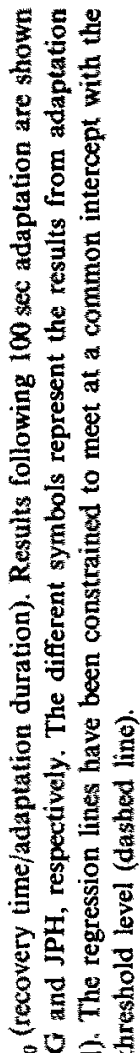
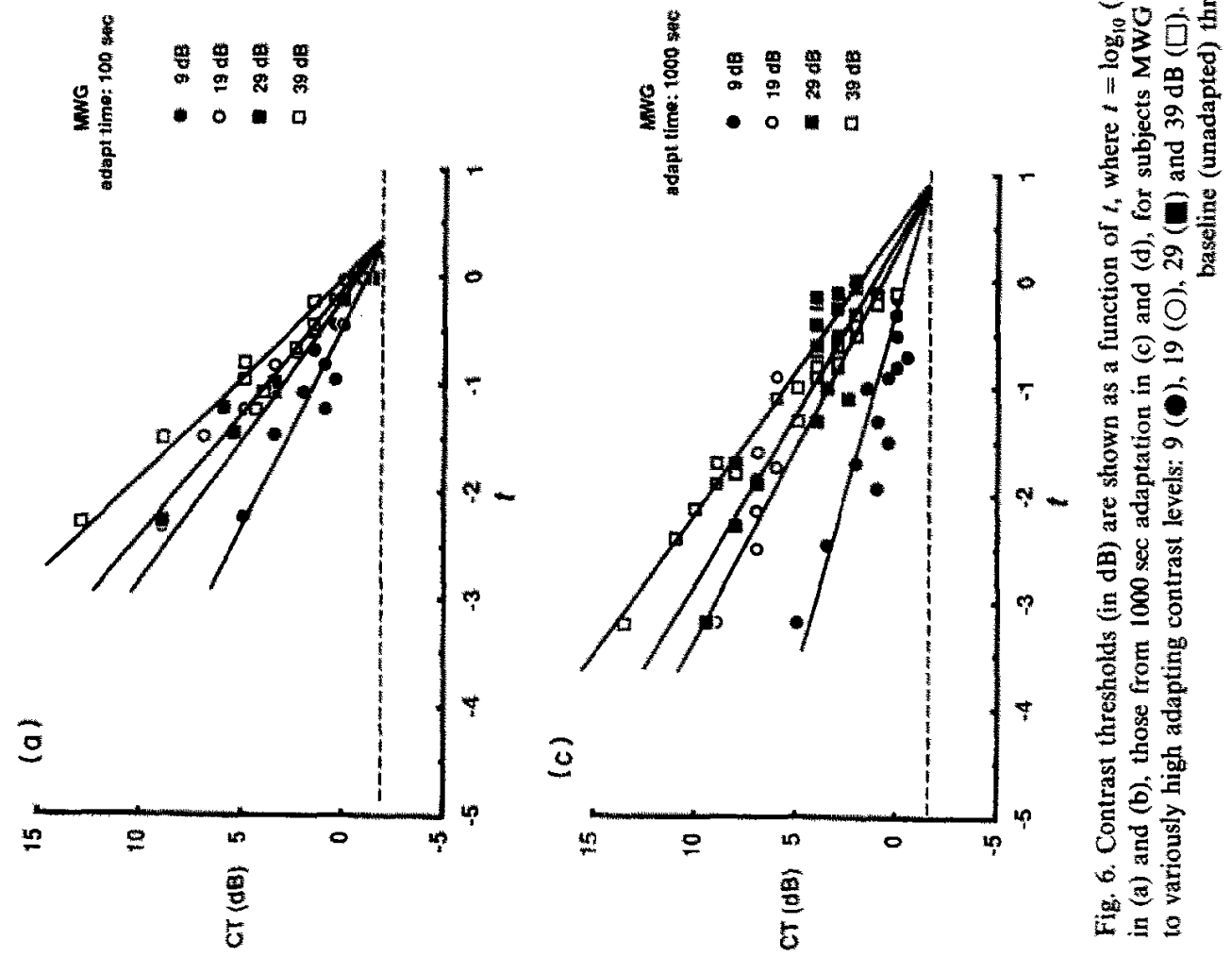

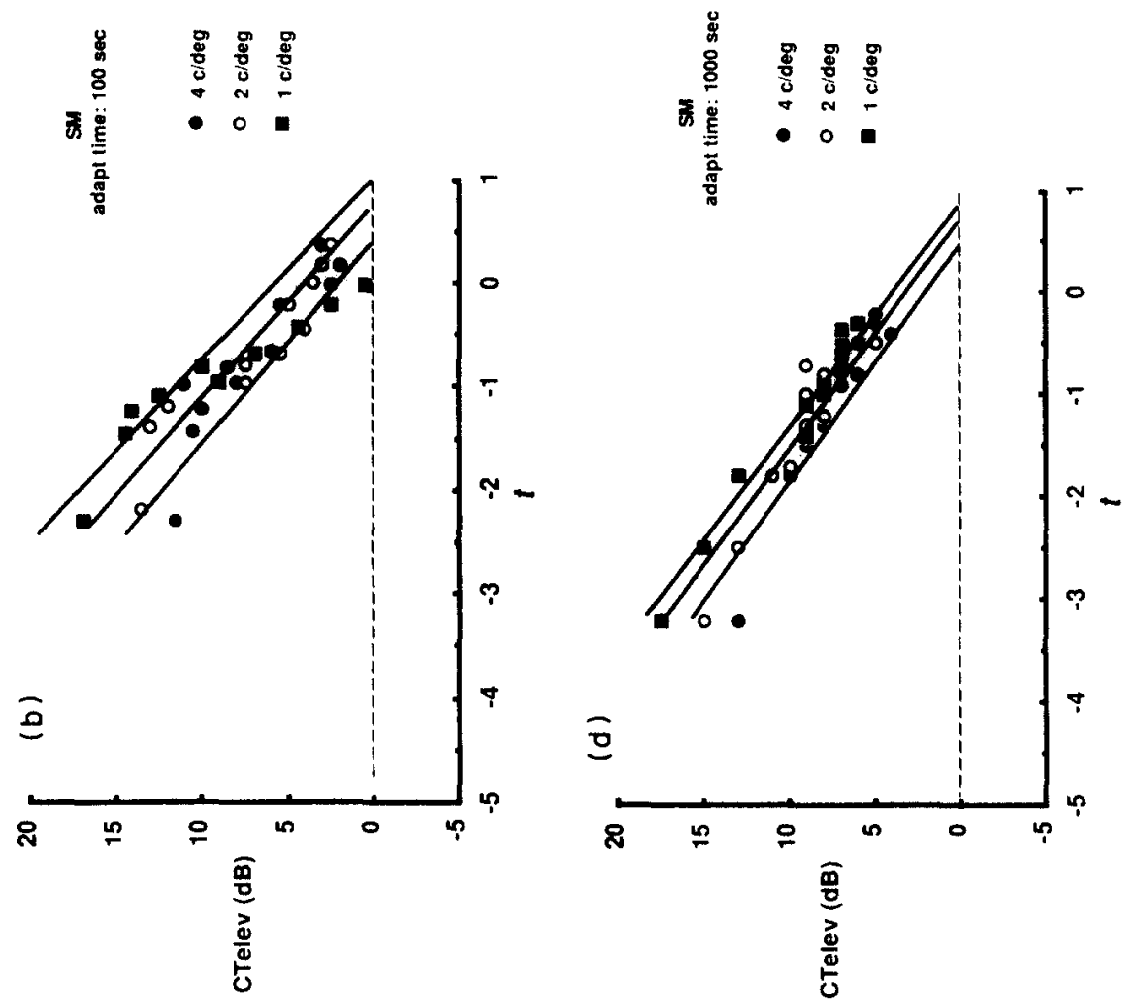

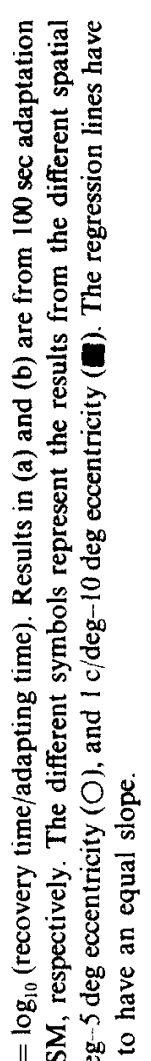
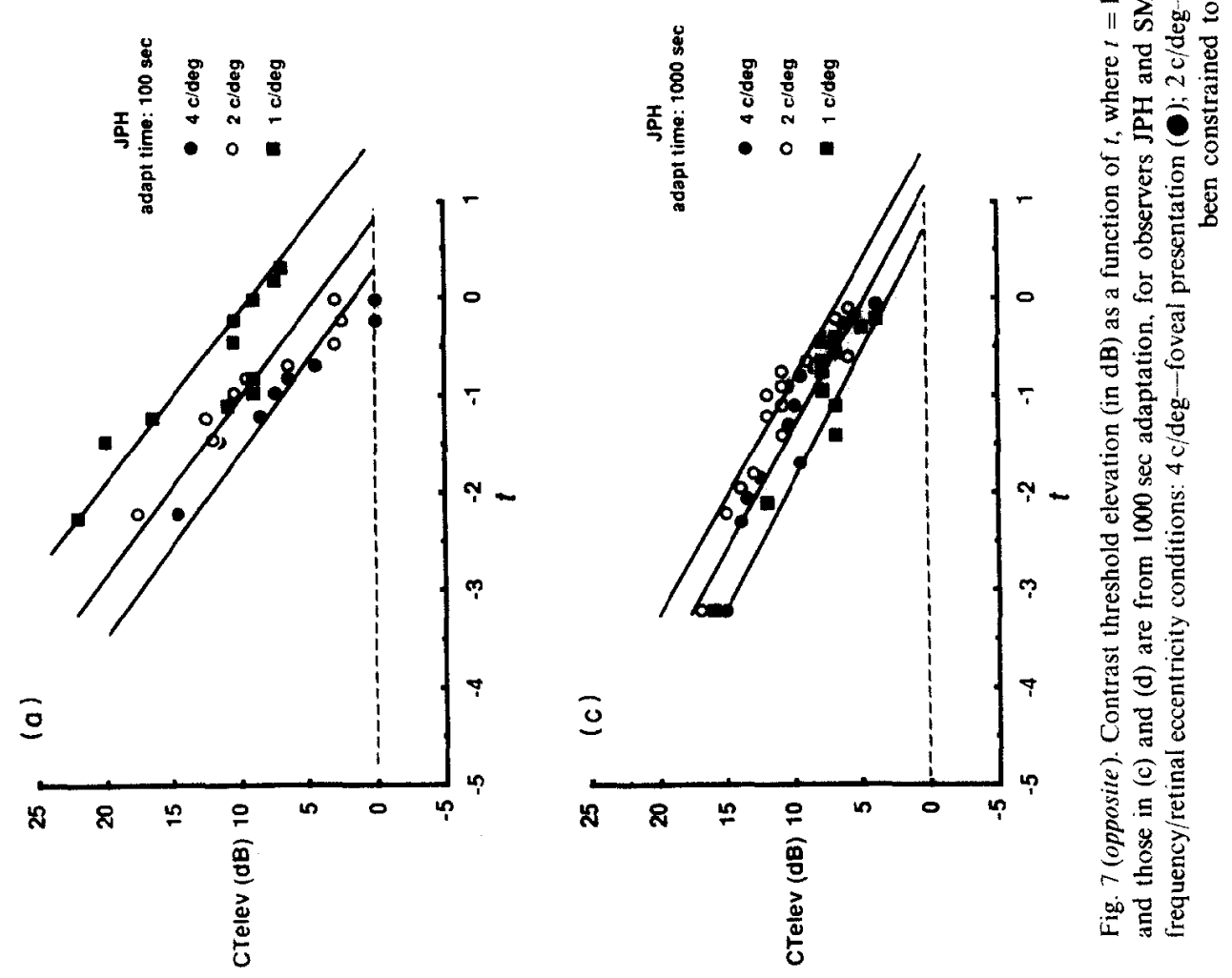
subjects either fixated in the middle of the screen (for the 0 and 5 deg eccentric test conditions) or $5 \mathrm{deg}$ right of the center of the screen (for the $10 \mathrm{deg}$ eccentric condition). In both cases, the adapting grating was large enough to subtend the entire central and near-peripheral visual field. The spatial frequency of the adapting and test grating was $4 \mathrm{c} / \mathrm{deg}$ for central testing, $2 \mathrm{c} / \mathrm{deg}$ for the $5 \mathrm{deg}$ test condition and $1 \mathrm{c} / \mathrm{deg}$ for the $10 \mathrm{deg}$ test condition. The width of the Gaussian envelope was adjusted so that each test grating had the same signal bandwidth ( 0.46 octaves). Adapting contrast was $39 \mathrm{~dB}$ in all conditions and the temporal frequency of the counterphased adapting and test gratings was $5 \mathrm{~Hz}$.

The results of these experiments are shown in Fig. 7 for observers JPH (a and c) and SM (b and d). Panels (a) and (b) present the results for the $100 \mathrm{sec}$ adapt duration and panels (c) and (d) show the results for the $1000 \mathrm{sec}$ adapt duration. Contrast threshold elevation is shown as a function of $t$ (cf. equation 2). The different symbols represent the results from the different frequency/eccentricity conditions: $4 \mathrm{c} / \mathrm{deg}$, foveal presentation ( $), 2 \mathrm{c} / \mathrm{deg}, 5 \mathrm{deg}$ eccentric presentation $(O)$, and $1 \mathrm{c} / \mathrm{deg}, 10 \mathrm{deg}$ eccentric presentation ( $\boldsymbol{D})$. Note that the regression lines have been constrained to have an equal slope. For the $100 \mathrm{sec}$ adaptation duration (Fig. 6a, b), there is a tendency for threshold elevation to increase with increasing retinal eccentricity. This tendency is more pronounced for subject JPH than for subject SM. This segregation for different retinal eccentricities becomes, however, for both subjects less clear for the $1000 \mathrm{sec}$ adaptation duration (Fig. $7 \mathrm{c}, \mathrm{d}$ ).

\section{DISCUSSION}

\section{The time course of recovery from adaptation}

The present findings indicate that recovery from contrast adaptation can best be represented as a power function of recovery time, yielding a linear fit on $\log -\log$ axes. On $\log$ threshold vs log recovery time axes, for a constant adapting contrast the slope of recovery from adaptation can be represented by a single exponent across different adapting durations. The decay constant corresponds to a decline of 4-6 dB per log unit recovery time (in sec) following adaptation to a grating having a $39 \mathrm{~dB}$ contrast level. Thus, it might be concluded from these results that recovery from adaptation is invariant across adapting times. Longer adapting times lead to larger threshold elevations that require more time to dissipate. But it is also clear from these results that knowledge of threshold elevation alone is not enough to specify the state of the underlying neurons. For example, as is evident in Fig. 2, a $10 \mathrm{~dB}$ elevation in threshold measured $1 \mathrm{sec}$ after $10 \mathrm{sec}$ adaptation required little more than $30 \mathrm{sec}$ to dissipate, whereas a $10 \mathrm{~dB}$ rise in threshold measured $10 \mathrm{sec}$ after $1000 \mathrm{sec}$ adaptation requires over $1000 \mathrm{sec}$ to return to baseline values. Interestingly, when we explored the effect of adapting contrast on the buildup and decay of adaptation (Figs 4 and 5) we found the different decay slopes to converge near the baseline value. Taken together, the results in Figs 3 and 5 suggest that the time to complete recovery does not depend on the initial threshold elevation, but rather on the time spent adapting, while the slope of the recovery function depends on the adapting contrast, but not on the adapting time.

The findings presented in Fig. 3 indicate that the recovery from adaptation to a high-contrast ( $39 \mathrm{~dB})$ grating can be depicted by a single function, if log contrast threshold is plotted as a function of $t$, where $t=\log _{10}$ (recovery time/ adaptation duration). Thus, for a constant adapt* ing contrast, the threshold elevation measured at $10 \mathrm{sec}$ after $100 \mathrm{sec}$ adaptation is equivalent to that measured at $1 \mathrm{sec}$ following $10 \mathrm{sec}$ adaptation or $100 \mathrm{sec}$ after $1000 \mathrm{sec}$ adaptation. The entire recovery process is merely shifted along the log time scale. Magnussen and Greenlee (1985) have shown, however, that this principle only holds for adaptation occurring within the dynamic range of the contrast-response mechanism. Adaptation lasting for $3 \mathrm{hr}\left(1.08 \times 10^{4}\right.$ $\mathrm{sec}$ ) dissipated in the same amount of time as did adaptation occurring at the point of the saturation of threshold elevation $\left(1.8 \times 10^{3} \mathrm{sec}\right)$.

By constraining the regression lines in Fig. 2 we assume that the recovery from adaptation can be described by a common slope, independent of the adaptation time. However, the statistical analysis of the effects of adapting time and adapting contrast on thresholds indicated that both factors significantly affect the resultant threshold elevation, as well as the recovery from adaptation. Compared to the effect of adapting time, the effect of adapting contrast yielded, however, larger $F$-ratios with respect to accounting for the variance of the slope of recovery. This result suggests that the slope of the recovery process is more strongly influenced by the contrast of the adapting stimulus, whereas the 
adaptation time determines, for the most part, how long the after-effect will persist.

\section{Effect of adapting contrast and duration on buildup of adaptation}

In Figs 4 and 5 we compared the effects of adapting contrast and adapting duration on the initial rise in thresholds. We estimated threshold elevation I sec after the cessation of adaptation by obtaining least-squares fitted regression lines between contrast threshold and recovery time. In Fig. 4 the buildup of adaptation with increasing adapting contrast corresponds to $4-6 \mathrm{~dB}$ per $\log$ unit adapting contrast. It is thus indicative of an exponent varying between 0.2 and 0.3 . In Fig. 5 we replotted the data shown in Fig. 4 to illustrate the buildup of adaptation with increasing adapting duration. This analysis yielded slopes corresponding to $4-6 \mathrm{~dB}$ per $\log$ unit adaptation time (in sec). Interestingly, the slope of the functions describing contrast threshold and adapting contrast is independent of the adapting duration (Fig. 4), and in similar fashion, the slope of the functions describing contrast threshold and adaptation duration is independent of adapting contrast (Fig. 5). A simple fatigue model of adaptation would imply that the slope of these functions should depend on the adapting contrast, since a higher contrast would fatigue the underlying neurons more rapidly. Our results clearly speak against such a relationship.

\section{Effect of retinal eccentricity on adaptation}

In Fig. 7 we compared recovery from adaptation for a centrally presented, $4 \mathrm{c} / \mathrm{deg}$ test grating to that found when a 2 or $1 \mathrm{c} / \mathrm{deg}$ test grating was presented at 5 or $10 \mathrm{deg}$ eccentric of fixation. The results of these comparisons indicated that the regression lines for the different adapting and test conditions did not converge, as in Fig. 6, in which are plotted data from the same adapting time but different contrasts. Rather, the lines were parallel, as in Fig. 2, which shows the effects of different adapting durations with a constant adapting contrast. This suggests that one of the effects of moving the adapting stimulus further into the periphery is more like increasing the adapting time for the foveal stimulus than increasing its contrast. This effect was, however, only pronounced for subject JPH after $100 \mathrm{sec}$ adaptation (Fig. 7a). The effect of retinal eccentricity was less clearcut for subject SM after $100 \mathrm{sec}$ (Fig. 7b) and after long adaptation times in both subjects $(1000 \mathrm{sec}$,
Fig. $7 \mathrm{c}$ and d). These observations suggest that the adaptation-induced threshold elevation in the peripheral visual field may saturate earlier compared to adaptation in the central foveal region. The findings, at least for shorter adaptation times, might also reflect higher gain in the peripheral channels, perhaps as a way to compensate for lower absolute sensitivity. Such increases in gain for low contrasts would serve to keep perceived contrast constant across the visual field despite variations in contrast thresholds (Georgeson \& Sullivan, 1975).

\section{Contrast gain control and neural memory}

The present results are also of interest with respect to the functional role of contrast adaptation. If adaptation were a mere reflection of neural fatigue (Swift \& Smith, 1982), that is to say that adaptation is dependent on the magnitude of prior activity in a neuron or "channel", then threshold elevation should show reciprocity on log adapt contrast-linear adapt time scales. Thus, within reasonable limits of temporal integration, there should be some other adapting contrast and time that could yield the exact same course of recovery as say $100 \mathrm{sec}$ adaptation to $19 \mathrm{~dB}$. The results in Fig. 6 show, however, that this is not the case. To predict the time required for recovery one needs to know how long the subject adapted and not at what contrast level. This is demonstrated in Fig. 8 in which are compared the results of earlier studies and the present findings. Estimated log recovery time is plotted as a function of $\log$ adaptation duration. The results from Magnussen and Greenlee (1985, subject RH), Björklund and Magnussen (1981), Lorenceau (1987), Rose and Evans (1983) and Heggelund and Hohmann (1976) are shown for comparsion. The regression of $\log$ recovery time on log adaptation time yielded a slope of 0.859 and an intercept of $0.622\left(r_{2}=\right.$ $0.941)$. The slope parameter does not significantly differ from unity at the 0.01 probability level, suggesting that the proportionality rule holds within this range. This proportionality rule also appears to hold for the suprathreshold appearance of the orientation of lines or gratings following adaptation, as the buildup and decay of the tilt aftereffect show similar time courses (Magnussen \& Johnsen, 1986; Greenlee \& Magnussen, 1987). Thus, contrast adaptation appears to be like a short-term memory store where the effects of adaptation persist for a length of time proportional to the adapting time. 


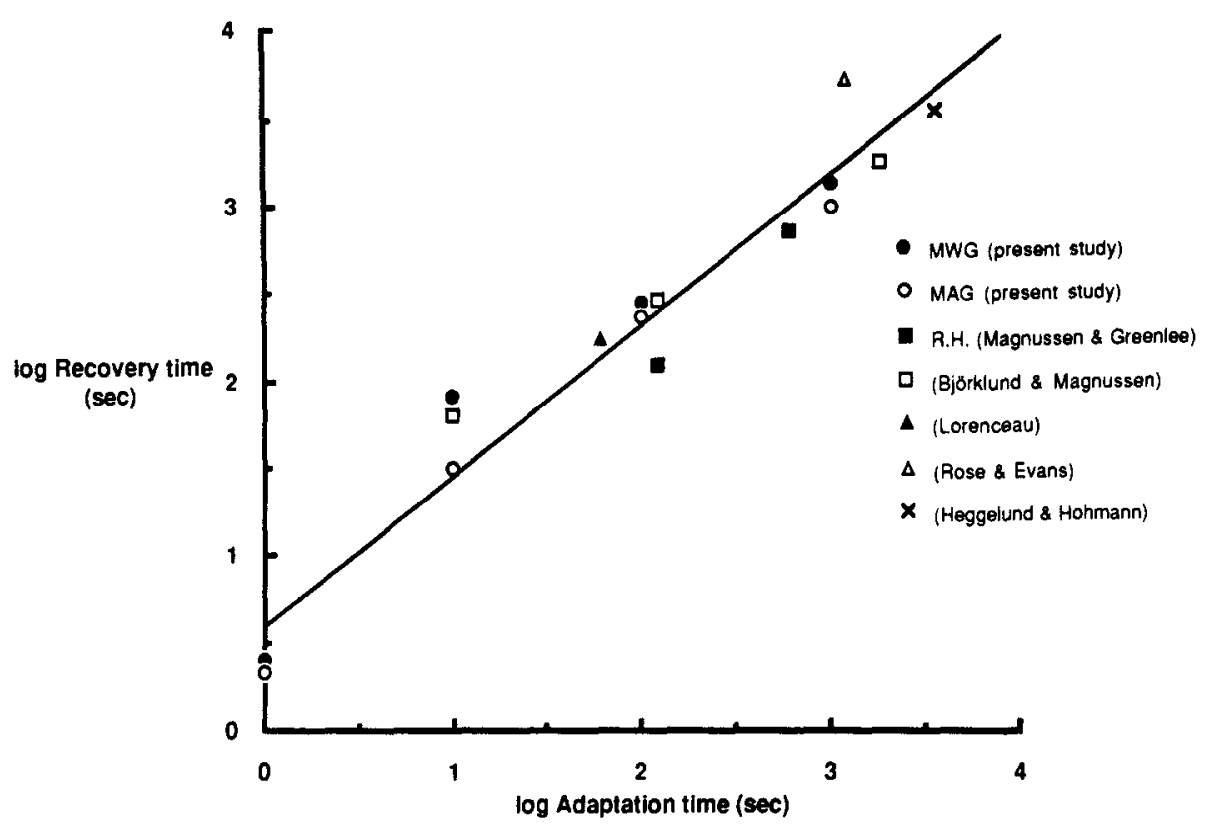

Fig. 8. Estimated log recovery time is plotted as a function of log adapting time. The different symbols are explained in the inset and show the results of various conditions conducted in the present study and those of Magnussen and Greenlee (1985), Björklund and Magnussen (1981), Lorenceau (1987), Rose and Evans (1983) and Heggelund and Hohmann (1976).

What might be the functional role of these adaptation processes? There is neurophysiological evidence in cat (Ohzawa, Sclar \& Freeman, 1985) and monkey (Sclar, Lennie \& DePriest, 1989) that cortical cells can adjust their gain to the prevailing contrast level. In an earlier study of human contrast adaptation Greenlee and Heitger (1988) showed that, after adaptation to a high contrast grating, contrast discrimination improved for gratings of high contrast. Such an improvement would be expected if a compressive contrast response function shifted its operating range, and so increased the slope of the function in the otherwise compressive range. It would clearly be useful if such a system responded quickly to the fast changes in contrast which would be encountered as one moved around in natural environments. Such fast changes are evident in our results for short adaptation times. However, it is not clear from this model why the recovery from adaptation should depend on the adaptation time, especially evident in the results from long adaptation times. Another functional role of contrast gain control in visual channels might be the self-calibration of the visual system. If a visual dimension such as orientation is coded by a number of overlapping channels, the activity of these channels might vary due to errors in the visual system itself, such as defects in the optics of the eye. Given some assumptions about the statistical properties of images (for example, that over time, the average activity in all orientation channels should be roughly equal), the gain of individual channels could be adjusted until these assumptions were met. When the visual input is highly selective over a prolonged period of time, as is the case for adaptation to a single sinewave grating, the rise in the activity, and the persistence of this enhanced activity of this channel would lead to a reduction in its gain and thus to subsequent aftereffects. This idea implies that each visual channel should carry a "record" of its own past time-averaged activity. Therefore, our present results and those of earlier studies are consistent with the idea of two functional roles for adaptation to spatial contrast: one is the short-term change in gain which shifts the operating range of the channel into the prevailing range of contrasts to maximize contrast resolution, the other is a long-term, "house-keeping" role, which compensates for the effects of constant errors, or drifts, in the performance of visual channels.

In summary, we have shown that the long recovery functions reported by Magnussen and Greenlee (1985) and others are not an artifact of the adjustment method. Within the dynamic range of the contrast gain control mechanism, the buildup and recovery from adaptation is 
best represented by a power function of time. Increasing adapting contrast increases the initial threshold elevation (estimated at $1 \mathrm{sec}$ recovery time using the best-fitting regression lines) but does not significantly increase the time required to recover from adaptation. The adaptability of mechanisms processing spatial contrast from the peripheral retinal appears to be slightly enhanced over those for central vision. We suggest that contrast adaptation reflects the combined effects of a dynamic contrast gain control mechanism and self-calibration in the visual cortex, and that the recovery from adaptation is a form of neural storage of recent activity occurring at the primary sensory encoding stage.

Acknowledgements - - This research has been supported by the Deutsche Forschungsgemeinschaft, SFB 325, B4, which also provided support to MAG and JPH during their stay in Freiburg. SM was supported by the Alexander von Humboldt Foundation. The authors would like to thank L. Spillmann for his hospitality and critical comments.

\section{REFERENCES}

Björklund, R. A. \& Magnussen, S. (1981). A study of the interocular transfer of spatial adaptation. Perception, 10, $511-518$

Blakemore, C. \& Campbell, F. W. (1969). On the existence of neurons in the human visual system selectively sensitive to the orientation and size of retinal images. Journal of Physiology, London, 203, 237-260.

Blakemore, C. \& Nachmias, J. (1971). The orientation specificity of two visual after-effects. Journal of Physiology, London, 213, 157-174.

Cowey, A. \& Rolls, E. T. (1974). Cortical magnification and its relation to visual acuity, Experimental Brain Research, 2I, 447-454.

Daugman, J. G. (1983). Dynamics of spatial channel adaptation. Unpublished doctoral dissertation, Harvard University, Boston, MA.

Georgeson, M. A. \& Georgeson, J. M. (1987). Facilitation and masking of briefly presented gratings: Time course and contrast dependence. Vision Research, 27, 369-379.

Georgeson, M. A. \& Harris, M. G. (1984). Spatial selectivity of contrast adaptation: Models and data. Vision Research, 24. $729-741$.
Georgeson, M. A. \& Sullivan, G. D. (1975). Contrast constancy: Deblurring in human vision by spatial frequency channels. Journal of Physiology, London, 252, 627-656.

Greenlee, M. W. \& Heitger, F. (1988). The functional role of contrast adaptation. Vision Research, 28, 791-797.

Greenlee, M. W. \& Magnussen, S. (1987). Saturation of the tilt aftereffect. Vision Research, 27, 1041-1043.

Heggelund, P. \& Hohmann, A. (1976). Long-term retention of the "Gilinsky-effect". Vision Research, 16, 10151017.

Ho, W. A. \& Berkley, M. A. (1988). Evoked potential estimates of the time course of adaptation and recovery to counterphase gratings. Vision Research, 28, 12871296.

Kulikowski, J. J., Marcelja, S. \& Bishop, P. O. (1982). Theory of spatial position and spatial frequency relations in the receptive fields of simple cells in the visual cortex. Biological Cybernetics, 43, 187-198.

Lorenceau, J. (1987). Recovery from contrast adaptation: Effects of spatial and temporal frequency. Vision Research, 27, 2185-2191.

Magnussen, S. \& Greenlee, M. W. (1985). Marathon adaptation to spatial contrast: Saturation in sight. Vision Research, 25, 1409-1411.

Magnussen, S. \& Johnsen, T. (1986). Temporal aspects of spatial adaptation: A study of the tilt aftereffect. Vision Research, 26, 673-675.

Ohzawa, I., Sclar, G. \& Freeman, R. D. (1985). Contrast gain control in the cat's visual system. Journal of Neurophysiology, 54, 651-667.

Pantle, A. \& Sekuler, R. (1968). Size-detecting mechanisms in human vision. Science, 162, 1146-1148.

Rose, D. \& Evans, R. (1983). Evidence against saturation of contrast adaptation in the human visual system. Perception and Psychophysics, 34, 158-160.

Rovamo, J. \& Virsu, V. (1979). An estimation and application of the human cortical magnification factor. Experimental Brain Research, 37, 495-510.

Sclar, G., Lennie, P. \& DePriest, D. D. (1989). Contrast adaptation in striate cortex of macaque. Vision Research, 29, 747-755.

Sekuler, R. W. \& Ganz, L. (1963). Aftereffect of seen motion with a stabilized retinal image. Science, 139, 419-420.

Swift, D. J. \& Smith, R. A. (1982). An action spectrum for spatial-frequency adaptation. Vision Research, 22, 235-246.

Watson, A. B. \& Pelli, D. (1983). QUEST: A Bayesian adaptive psychometric method. Perception and Psychophysics, 33, 113-120.

Williams, D. W. \& Wilson, H. R. (1983). Spatial-frequency adaptation affects spatial-probability summation. Journal of the Optical Society of America, 73, 1367-1371. 